\title{
Vision Disorder
}

National Cancer Institute

\section{Source}

National Cancer Institute. Vision Disorder. NCI Thesaurus. Code C35126.

Any impairment to the vision. 